H I G H L I G H T S

\title{
Cues for germination
}

In you or me, the path from fertilized egg to mature embryo is relatively smooth and predictable. By contrast, plant embryonic development is fitted with a safety catch: seeds can remain dormant until a combination of intrinsic and extrinsic factors triggers them to germinate. Environmental cues, which might be temperature or moisture, are well documented, as are the intrinsic signals, such as the plant hormone gibberellin (GA), with which they interact in the seed. But, until now, a direct molecular connection between the two had not been found. Reporting in Genes and Development, Lee and colleagues have found that the Arabidopsis RGL2 gene - which encodes a putative transcription factor - acts at the interface between imbibition (water uptake by the seed) and signalling by GA.

The germination of Arabidopsis seeds begins when, under favourable moisture conditions, GA reaches a critical level. How GA regulates germination is not understood, but genetic analysis indicates that neither GA nor the proteins that mediate GA signalling directly link moisture to germination. Reasoning that the crucial integrator probably resembles these proteins, Lee et al focused on several uncharacterized genes that encode functional domains related to those in some GA signalling molecules. Genetic epistasis analyses with the gene RGL2 - using null alleles that were recovered from a transposon integration database - were particularly striking. Mutations in RGL2 completely restored germination to plants that were genetically or chemically deficient for GA signalling. This implies that RGL2 is a negative regulator of GA responses - an effect that the authors found was specific to germination.

But how do moisture, GA signalling and RGL2 interact? A clue to this came from looking at $R G L 2$ expression: in the embryonic shoot of wild-type Arabidopsis, levels of $R G L 2$ mRNA rise on imbibition, then fall as the embryonic shoot breaks through the seed coat. RGL2's expression is therefore more or less directly induced by moisture. When GA is not around, RGL2 transcript levels stay high — although, significantly, they fall if GA is administered. By fitting this, and other information, into a model, Lee et al. propose that $R G L 2$ levels rise on imbibition, blocking germination until levels of GA are high enough to bring RGL2 levels back down.

This work describes the first direct molecular link between an environmental and an endogenous cue in seed germination. More genes and more studies will no doubt complete the picture, but this remains a successful example of how gene functions can be assigned in this 'post-genomic' organism.

Tanita Casci

6) References and links

ORIGINAL RESEARCH PAPER

Lee, S. et al. Gibberellin regulates Arabidopsis seed germination via RGL2, a GAV/RGA-like gene whose expression is up-

regulated following imbibition. Genes Dev. 16, 646-658 (2002) WEB SITES

Jinrong Peng's lab:

http://research.ima.org.sg/research/plant/pengir Nicholas Harberd's lab:

http://www.jic.bbsrc.ac.uk/staff/nicholas-harberd

\section{Introns branch out}

The origin of eukaryotic introns has been hotly debated since their discovery $\sim 25$ years ago. From this debate, two key theories have emerged to explain how introns came into existencethe introns-early hypothesis, which proposes that protein-coding genes existed in the last universal common ancestor (LUCA), and the introns-late hypothesis, which suggests that introns were absent from the LUCA and arose in eukaryotes. There is evidence to support both

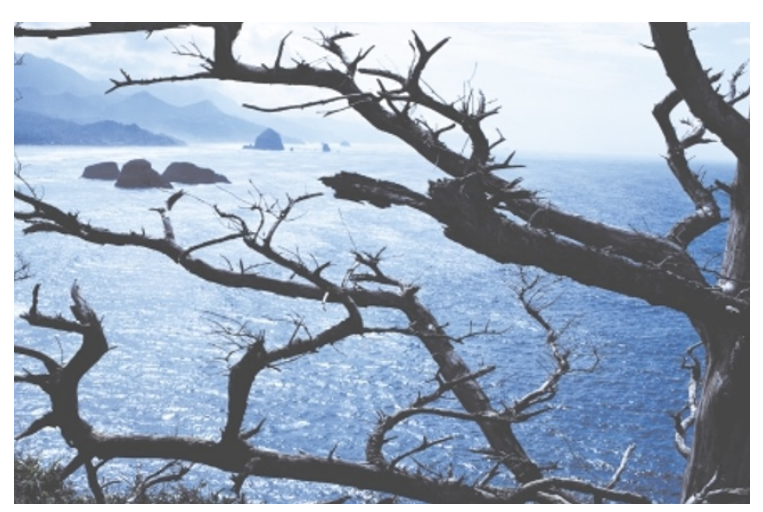

theories. In support of the introns-late hypothesis, for example, is that most eukaryotes have introns - that is, except for some ancestral single-celled eukaryotes (protists), such as Giardia lamblia, that lie in the deepest branches of the eukaroytic lineage. Now Julie Nixon and colleagues have discovered a G. lamblia gene that actually does contain at least one intron, as well as genes that encode the splicing machinery, which places the origin of introns quite a bit earlier than previously thought.

The authors made their discovery when they spotted an in-frame stop codon in a G. lamblia sequence that encodes the ironcarrying protein ferredoxin in the results of a BLAST search. Could this stop codon lie in an intron and be removed by splicing? To investigate this, Nixon et al. amplified the coding region of this gene by reverse transcription-PCR and isolated a spliced sequence from which a 35-bp intron had been removed. This intron contained a canonical 3' splice site, a non-canonical 5' site and a branch-point sequence similar to the consensus branch-point sequence in yeast.
Next, the authors looked for the machinery required to remove this intron by searching G. lamblia sequence for homologues of Saccharomyces cerevisiae's spliceosomal proteins. This search identified many putative G. lamblia homologues of such proteins, including homologues of Prp8 and Prpr11, which are eukaryote-specific spliceosomal peptides, six DexH-box RNA helicase homologues, which are involved in the splicing reaction and in releasing spliced products from the spliceosome, and homologues of two proteins that make up a splicing complex called Brr2.

So, what do these findings tell us about the origins of introns? If the introns-late theory is correct, then splicing probably arose much earlier than previously expected in eukaryotic evolution. However, as the authors point out, whether it's introns-early or introns-late is still up for debate.

Jane Alfred

\section{(1) References and links}

ORIGINAL RESEARCh PAPER Nixon, J. E. et al.

A spliceosomal intron in Giardia lamblia. Proc. Natl Acad. Sci. USA 2002 (10.1073/pnas.042700299)

WEB SITES

Encyclopedia of Life Sciences: http://www.els.net

Universal tree of life

Universal tree of life

http://www.hsph.harvard.edu/faculty/JohnSamuelson.html 